\section{$\underset{\substack{\text { hommes } \\ \text { \& migrations }}}{ }$}

\section{Hommes \& migrations}

Revue française de référence sur les dynamiques

migratoires

$1293 \mid 2011$

L'immigration dans les musées

\title{
La muséification de la migration à Berlin et les débats sur la représentation
}

\section{Andrea Meza Torres}

Traducteur : Françoise Bouillot

\section{(2) OpenEdition}

\section{Journals}

\section{Édition électronique}

URL : http://journals.openedition.org/hommesmigrations/498

DOI : 10.4000/hommesmigrations.498

ISSN : 2262-3353

Éditeur

Musée national de l'histoire de l'immigration

Édition imprimée

Date de publication : 1 septembre 2011

Pagination : 28-39

ISSN : 1142-852X

Référence électronique

Andrea Meza Torres, «La muséification de la migration à Berlin et les débats sur la représentation », Hommes \& migrations [En ligne], 1293 | 2011, mis en ligne le 31 décembre 2013, consulté le 19 avril 2019. URL : http://journals.openedition.org/hommesmigrations/498 ; DOI : 10.4000/ hommesmigrations.498 


\section{La muséification de la migration à Berlin et les débats sur la représentation}

Par Andrea Meza Torres, doctorante à l'Institut für Europäische Ethnologie, Humboldt Universität zu Berlin

Représenter la migration dans un musée pose la question de la distance face à ce qui est exposé : non plus seulement des œuvres, mais des traces de parcours de vie. Face aux clichés exotiques, coloniaux, ou simplement à la tentation d'une vision figée de l'altérité, les modalités de la représentation muséale de l'expérience migratoire apparaissent complexes car chaque décision est lourde de sens. Tout dépend de la négociation entre les attentes de l'institution et la capacité des migrants à se réapproprier le récit de leur propre histoire. 
La question de la muséification est l'un des multiples éléments d'une crise de la représentation dont l'un des points focaux a été les batailles théoriques qui se sont livrées aux États-Unis dans les années quatre-vingt ${ }^{(1)}$. Elles ont suscité de vifs débats dans tous les domaines concernant la représentation : la formation du savoir académique et l'enseignement des disciplines ${ }^{(2)}$; la pratique de l'écriture ethnographique $^{(3)}$; les débats sur les identités "nationales", la citoyenneté, la migration et les minorités ethniques, avec les mouvements des Chicanos et des Noirs et l'entrée des "decolonial studies" dans les cursus universitaires ${ }^{(4)}$. Ces débats ont fini par atteindre le domaine muséal et ont naturellement influencé la production des représentations ${ }^{(5)}$. Les paysages muséaux sont devenus d'importants domaines de recherche, car ils sont des arènes où la crise de la nation se confronte aux demandes de représentation sociale des minorités - migrantes ou non - et des diasporas, et aux questions soulevées par les études post-coloniales et décoloniales. Les expositions berlinoises sur la migration illustrent parfaitement la façon dont cette crise est devenue visible, témoignant de tous les débats que peut susciter le musée sur la création d'images de l'Europe et d'une nouvelle politique de migration. Les trois cas ethnographiques présentés ici, à partir de trois exemples de muséification de la migration à Berlin, sont issus d'une recherche menée de juillet 2009 à octobre 2010. Des entretiens ont été réalisés avec les acteurs des musées et des expositions (commissaires et migrants ou groupes de migrants) en vue de distinguer des arènes de conflits pertinentes où apparaisse clairement cette crise de la représentation. Les cas présentés peuvent être regardés comme des "objets" ayant émergé de l'interaction avec le domaine de la migration et ses acteurs dans les musées. Ces "objets" sont liés aux espaces où est contestée la formation des savoirs établis.

En effet, les peuples, les migrants et leurs "corps" jouent un rôle central dans les représentations de la migration - que ce soit comme objets, comme acteurs, ou encore en tant que personnel et commissaires dans le musée -, dissolvant ainsi la limite entre des notions établies de "soi" et des

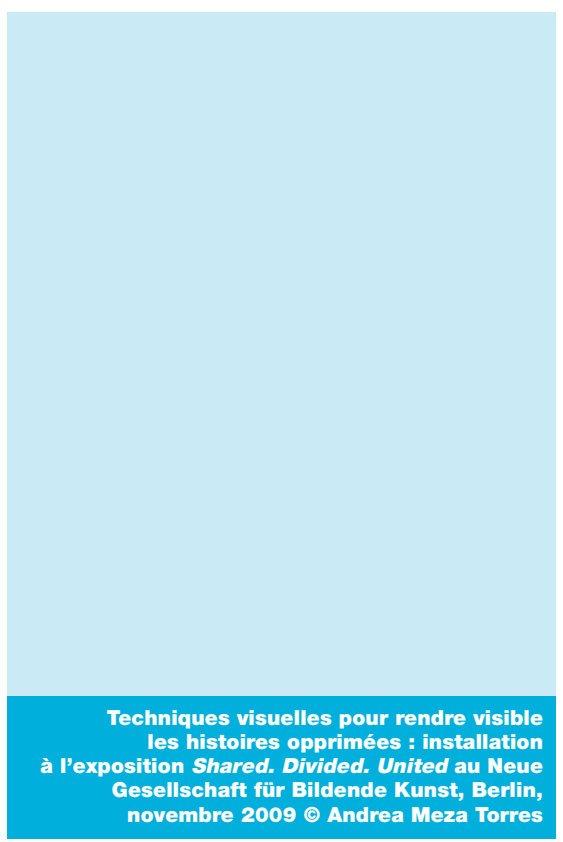


“autres". Les trois espaces décrits à Berlin sont en réalité très disséminés, car Berlin n'a pas d'espace centralisé pour exposer la migration. Dans ces lieux, le rapport entre les corps, les objets et les scènes muséales se fait plus tendu. La figure de l'immigré en tant que personne représentée dans le musée suscite bien des questions sur les représentations. La distance entre les images et les personnes représentées tend à disparaître, ce qui veut dire que les représentations peuvent être directement contestées à tout moment.

\section{Les migrants, acteurs du musée}

Le premier exemple est un petit musée de Berlin, le Jugendmuseum de Schöneberg, qui s'adresse aux enfants et aux jeunes dans le quartier de Tempelhof-Schöneberg. Il a pour objectif de représenter l'histoire et la société contemporaines du quartier, dans le cadre d'un complexe muséal local constitué par deux autres petits musées (Stadtteilmuseen). C'est là qu'est installée depuis 2002 l'exposition Villa Global qui vise à montrer la diversité culturelle des habitants du quartier. Cette exposition a été choisie pour sa façon d'engager la communauté du quartier dans ses pratiques curatoriales. Conçue à partir de la pédagogie muséale, du travail social et du dialogue interculturel, elle a ouvert un petit espace théâtral et social où la question de l'altérité a acquis toute sa dimension.

Villa Global représente un bâtiment de 14 chambres occupées par des gens d'origines différentes qui résident dans le quartier de Tempelhof-Schöneberg. Pour organiser l'exposition, le musée a donc travaillé avec de "véritables voisins". Le directeur et le personnel ont choisi des gens "issus de l'immigration". Les participants ont conçu librement leur propre chambre, en choisissant thèmes, objets et stratégies de représentation de leur choix, chaque participant réalisant sa propre installation. Cette démarche a suscité des questions fondamentales quant à leur participation au musée. Cette complexité s'est encore accrue lorsque certains commissaires ont été intégrés au musée à titre de guides de Villa Global.

Lors d'une visite au musée au printemps 2010, j'ai voulu en savoir davantage sur les effets d'"auto-exotisation" que suscitaient certaines chambres. Ainsi, dans la chambre "péruvienne" de M. Rodriguez, je me suis trouvée face à de nombreuses images du Macchu Picchu accrochées au mur. La pièce était remplie de symboles péruviens et latino-américains, comme une photo de Che Guevara, des CD de salsa et de rythmes afro, ainsi qu'un autel baroque honorant plusieurs saints. Cette chambre ressemblait plus à un musée qu'à un lieu de vie. À côté, la chambre de M. Odgesou présentait elle aussi beaucoup d'éléments traditionnels, mais le visiteur 
pouvait au moins s'installer dans un canapé confortable pour regarder une série télévisée ghanéenne.

Au cours de mon périple dans l'hôtel, j'ai demandé à la commissaire d'exposition qui s'était chargé de quelle chambre. Elle m'a répondu : "Eh bien, pas mal de gens... Moi, par exemple." Mme Dubinina s'était chargée de la chambre "ukrainienne". Quand elle m'a montré ses objets, j'ai eu l'impression de me trouver dans sa propre chambre. Nous avons pris le téléphone et écouté une conversation dans sa langue maternelle. Elle a ensuite expliqué où elle avait acheté chaque objet, elle a raconté les histoires auxquelles ils étaient rattachés et ce qui l'avait poussée à les emporter avec elle jusqu'à Berlin. Comme nous sortions de la pièce, je lui demandai si je pouvais voir quelqu'un d'autre. On croisa un homme qui se révéla être le curateur de la chambre "iranienne/persane". Il était revenu au musée pour vérifier et remplacer certains objets. Nous sommes allés dans sa chambre, elle aussi remplie d'objets très traditionnels, qui n'auraient pas déparé dans un musée ethnographique. M. Bahadoran a réalisé une performance avec certains des objets tandis que nous parlions de révolution et d'exil.

\section{De la personnification au risque de l'exotisme}

Chaque chambre avait un nom propre. Tous étaient des pseudonymes, sauf un : Layla, qui travaillait pour le musée pendant les week-ends. Lors de ma visite suivante, Layla se tenait dans le hall où elle accueillait les visiteurs avec son voile sa tenue habituelle. Elle ne faisait pas semblant d'être quelqu'un d'autre. Elle me fit visiter l'exposition et surtout sa chambre, qui était une sphère très intime, très élégamment décorée, où elle avait exposé l'histoire de son mariage et les images de ses noces. Elle me montra une collection de voiles qu'elle allait souvent présenter aux enfants des écoles et toutes ses photos de mariage.

Après quoi, elle m'emmena dans la chambre de Yücel. Cette chambre était un lieu "turc" très traditionnel et en même temps très réel. Elle comportait un petit salon de thé, un lit, et les objets et les images du rituel de circoncision de son propre fils. Après la visite, Layla accepta de me mettre en contact avec Yüicel. J'allai lui rendre visite dans sa boutique, dans un autre quartier de Berlin. Là, nous avons discuté un bon moment de l'exposition de l'intimité et d'autres sujets. Elle me confia notamment que son propre fils, quelques années après l'ouverture de l'exposition, l'avait priée de démonter le rituel de circoncision, qui devenait trop intime pour lui à présent qu'il était adulte. Nous discutâmes ensuite de la façon dont les immigrés 
Des effets personnels dans la chambre ukrainienne, au Jugendmuseum de Schöneberg, Berlin, avril $2010 \odot$ Andrea Meza Torres développent plusieurs personnalités. Celle qu'elle avait laissée dans la chambre du musée n'était qu'un aspect d'elle-même ; c'était son moi traditionnel, par le biais duquel elle vivait certains aspects de sa vie. Mais cette image n'épuisait pas toute sa personnalité. C'est pourquoi elle avait cette boutique, qui offrait une image moderne d'une femme indépendante. Mais cela aussi n'était qu'un aspect parmi d'autres. Quand on l'avait priée de réaliser une chambre pour le musée, elle avait pensé que le mieux serait de montrer une version compacte d'ellemême - une collection de son moi "traditionnel". Ce voyage au musée m'a menée au plus près de l'intimité des gens, tout en m'entraînant à l'autre bout de Berlin. Entrée dans le musée à Schöneberg, j'en suis ressortie dans le quartier de Wilmersdorf-Charlottenburg. J'ai été très surprise de ce voyage qui avait commencé par une prise de contact avec une exposition du réel. La présence corporelle des concepteurs dans leur propre chambre ouvre une zone de contact, un espace de performance entre les sphères de curation, les objets représentés, les processus d'apprentissage et la vie quotidienne. C'est une scène en mouvement constant.

Au cours d'un entretien en 2010 avec la directrice du musée, Petra Zwaka, nous avons discuté des risques et des avantages de ce genre de mises en scène. Le risque d'auto-exotisation et de surexposition de l'intimité pourrait devenir problématique, car les concepteurs peuvent aisément perdre le sens de la limite quant aux objets à exposer - induisant ainsi un effet de "carnaval". Un autre problème était le fossé générationnel. Alors que les vieilles générations tendaient à se concentrer sur la tradition, les jeunes montraient d'autres façons de représenter leurs souvenirs. C'était visible notamment dans la sélection des objets de la vie quotidienne : les anciens se distinguaient en exposant des meubles traditionnels, alors que les jeunes préféraient apporter des éléments Ikea. Cela générait une tension intergénérationnelle entre différentes façons d'exposer l'altérité et des images de soi. 
Zwaka avait déjà tenté d'introduire des changements dans l'exposition en priant de nouvelles personnes d'y entrer alors que d'autres en sortaient. Mais elle n'en était pas satisfaite et envisageait de nouvelles modifications ${ }^{(6)}$. L'exposition à la Jugendmuseum Schöneberg dure depuis plus de huit ans, et les représentations et performances ont un air un peu daté. Cette instabilité tient en partie au fossé générationnel mais aussi à la nature des expositions sur la migration qui doivent être en transformation constante pour faire sens. Les expositions sur la migration risquent d'avoir une vie assez brève, surtout quand elles sont associées à des communautés en transformation constante. Les changements dans l'identité et dans les rapports entre des espaces transnationaux signifient aussi des changements dans la représentation.

\section{Dépasser les imaginaires coloniaux}

Le second exemple est un projet du département africain de l'Ethnologisches Museum de Dahlem, issu d'un projet plus vaste conçu par Peter Junge, directeur du département, pour le futur Humboldt-Forum de Berlin". Berlin, capitale "en train de se faire", continue à réorganiser toute une gamme de représentations et de collections muséales autour de l'Humboldt-Forum, qui sera situé au cceur de la ville. Même si l'on sait que le projet du Humboldt-Forum n'envisage pas d'inclure le thème de la migration, celui-ci suinte de tous ses murs par le biais des acteurs, des corps et des objets. Le département africain du musée a développé un projet spécialement conçu pour le Humboldt-Forum. Il présente l'intérêt de travailler sur la notion de communauté, mais en abordant la question de la diaspora à Berlin. Il contraste avec d'autres départements du musée d'ethnologie de Dahlem, dont les projets pour le HumboldtForum concernent des communautés traditionnelles et ethniques vivant par exemple en Alaska ou au Mexique.

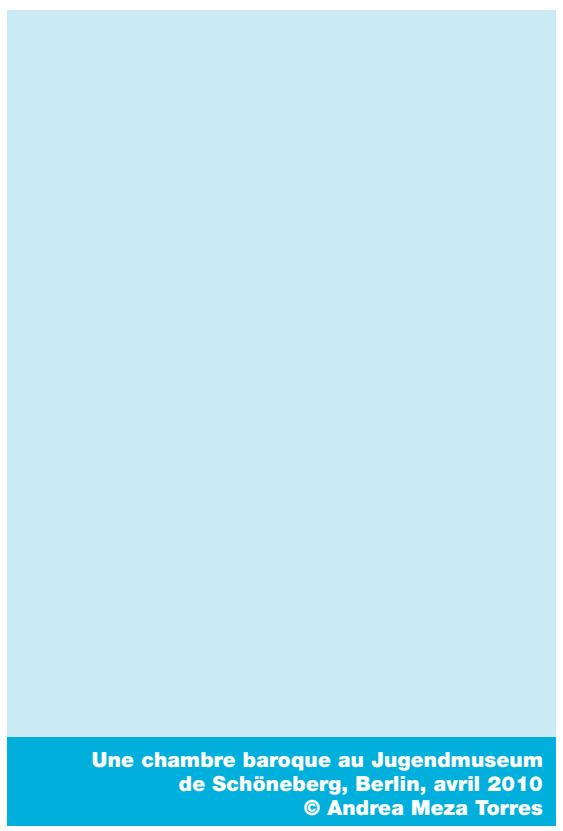


Le département africain semble travailler avec une communauté nigériane, mais en réalité ce sont des gens qui sont venus du Nigéria (leur lieu de naissance) à Berlin. En clair, des immigrés officiellement associés à Berlin et engagés dans la vie culturelle de la ville. Néanmoins, le musée ne veut pas les qualifier d'immigrés. Junge refuse explicitement d'opérer ce tournant, bien qu'il ait eu lui-même accès à une pièce très importante pour son projet dans un lieu traversé par les processus de migration : le Karneval der Kulturen ("Carnaval des cultures") de Berlin.

Depuis 2006, la transformation de l'héritage colonial du musée a été engagée en vue de l'inscrire dans le projet Humboldt-Forum : la première étape a consisté à présenter des objets ethnographiques comme de l'art (Kunst aus Afrikal" Art d'Afrique", 2006), et la seconde, à étendre le projet avec l'exposition Ijele. Zeitgenössische Kunst. Bamum. Benin ("Ijele. Art contemporain. Bamum. Bénin") en septembre 2009. Cette nouvelle étape commence par une petite pièce dans laquelle est exposé un gros objet coloré : le masque ijele. Celui-ci a été fabriqué au Nigéria spécialement pour le Carnaval des cultures de Berlin. Il est présenté comme un élément du travail interculturel de l'association Ikuku-Berlin ${ }^{(8)}$ pour le Carnaval. Pour aquérir ce masque, Junge avait négocié avec John Durumba, directeur de l'association nigériane Ikuku-Berlin. L'un et l'autre désiraient voir le masque exposé au musée. Tous deux ont été interrogés et il ne semble pas que les négociations aient suscité de fortes tensions. Lors d'une conversation en novembre 2009 avec John Durumba à Ikuku-Berlin, ce dernier a indiqué que Peter Junge avait vu le masque lors du Carnaval des cultures et qu'il les avait appelés trois mois plus tard. C'est ainsi qu'ils s'étaient rencontrés pour discuter de la présentation du masque au musée. Junge a donné une version similaire lors de son interview, le 22 avril 2010. Il a raconté comment il avait vu le masque ijele au Carnaval et avait voulu l'exposer au musée. Ce masque selon lui était une rareté, le seul exemplaire qu'il ait vu hors du Nigéria. Il avait été surpris. Peu de temps après le Carnaval, alors qu'il se demandait comment l'obtenir, il avait reçu un coup de fil d'Ikuku-Berlin.

\section{Déjouer les simulacres de l'altérité}

Limportant ici est la signification de l'institution du Carnaval des cultures à Berlin comme une zone de contact entre les musées et les organisations sociales migrantes. Le Carnaval était l'endroit où le masque était exposé pour un public berlinois - ce qui revient à dire que l'objet était déjà conçu pour un public spécifique. Et ce fut le Carnaval qui permit un contact rapide entre Ikuku-Berlin et le département africain de l'Ethnologisches Museum. Le Carnaval a donc joué un rôle de médiateur entre les deux partenaires. 
Néanmoins, l'ambivalence présidant à l'acquisition du masque est précisément ce qui pousse à contester la représentation d'Ikuku-Berlin comme une "diaspora". Le Carnaval est certes un site important de participation sociale et d'exhibition des "différences" culturelles. On estime que le Carnaval de Berlin est issu du Notting Hill Carnival de Londres. Le Karneval der Kulturen de Berlin incarne une sorte d'extension de type rhizome. D'autre part, c'est aussi un lieu d'auto-exotisations où des objets fabriqués à Berlin risquent d'être perçus comme relevant de "l'autre". Il offre notamment une plate-forme de collecte pour les conservateurs de musée. Le Carnaval est donc un marché du primitivisme qui maintient les représentations à la place stable de "l'altérité". Durant un long week-end de C'est aussi dans ce cadre que la culture nigériane peut être liée à l'héritage colonial de l'Allemagne, par le biais d'un objet. célébrations, il naturalise les participants et les objets en tant qu'“autres", et c'est dans cet espace liminal que la négociation des objets commence. Outre l'exemple du département africain de l'Ethnologisches Museum, nous pouvons citer celui du Stadtteilmuseum Neukölln qui montre un masque colombien acquis au carnaval dans sa nouvelle exposition. Et c'est aussi dans ce cadre que la culture nigériane peut être liée à l'héritage colonial de l'Allemagne - par le biais d'un objet.

Dans l'exemple du masque ijele, des gens qui vivent depuis longtemps en Allemagne sont présentés comme une diaspora et juxtaposés aux collections coloniales, faisant ainsi silence sur des processus de migrations qui peuvent émerger à tout moment ${ }^{(9)}$. Quand la migration gît dans la toile de fond d'un processus culturel, elle tend à imprégner toutes les représentations et à émerger aux marges des politiques ou des productions culturelles, même dans les contextes où elle n'est pas désirée. Elle peut déstabiliser l'ensemble des représentations. C'est d'ailleurs l'un des points sur lesquels le Humboldt-Forum a suscité la critique. Ainsi, l'ethnologue Beate Binder décrit comment son programme annonçait un "dialogue des cultures" avec une image d'une danseuse exotique au Karneval der Kulturen ${ }^{(10)}$. Il semble que, dans le projet Humboldt-Forum, ce soit le Carnaval qui présente le plus d'images de "l'altérité", il a donc acquis une importante visibilité à Berlin et en Allemagne. Les projets locaux/nationaux se sont approprié la dynamique de ce carnaval né dans les faubourgs de Londres. Le Carnaval des cultures pourrait ainsi avoir pour rôle de créer et de pérenniser un espace périphérique pour l'exposition de l'altérité, faisant de celui-ci un endroit assez stable pour y exécuter des performances "temporaires" où soient simulés des actes de participation.

C'est là un phénomène très important, parce qu'il peut être comparé à la dynamique du marché de l'art contemporain, florissant à Berlin depuis des années. 
Revenons un instant à l'exposition Ijele. Zeitgenössische Kunst. Bamum. Benin: une fois passée la petite pièce où est exposé le masque ijele, on parvient à un espace intitulé "Art contemporain/Afrique". On y perçoit un rapport évident entre le musée et le marché de l'art - les galeries et les biennales. Ces dernières présentent les images de l'altérité et mettent en contact les artistes et les musées ethnologiques. Dans les ceuvres que montre l'exposition, on ignore selon quels critères cet art est représentatif de "l'Afrique". Le marché de l'art est un processus par lequel l'art est présenté sous forme de musées, qui jouent donc un rôle similaire au Carnaval en simulant une participation d'“Africains" à l'exposition.

Mais il existe aussi à Berlin des institutions artistiques qui jouent un rôle important pour modifier ces dynamiques. Les institutions artistiques démocratiques offrent une scène où de nouveaux acteurs peuvent dépeindre dans leurs propres termes le travail de la communauté, les identités transnationales et la migration.

\section{Une réappropriation des décisions muséales}

L'exposition intitulée Partagés. Divisés. Unis, qui a eu lieu dans une institution artistique, la Neue Gesellschaft für Bildende Kunst (NGBK) ou "Nouvelle Société pour les arts plastiques ${ }^{(11)}$,", permet de questionner le rôle des espaces artistiques dans la représentation de la migration. Inaugurée en octobre 2009, elle incarnait la convergence de l'organisation immigrée germano-coréenne Korientation et de cette institution artistique, la NGBK, dont quasiment tout le monde peut devenir membre. La règle ici est qu'il faut l'appui de cinq membres du conseil des commissaires pour qu'un projet soit approuvé. Cinq membres de la "première" et de la "deuxième" générations, dont certains sont passés par l'université et se définissent eux-mêmes comme germano-coréens, ont intégré cette institution démocratique et ont conçu un concept d'exposition qui a été approuvé.

Partagés. Divisés. Unis était une exposition unique en termes de stratégie de représentation. La narration était construite à la façon d'une installation, car elle créait l'histoire à partir d'objets collectés auprès de gens ayant personnellement vécu une expérience de migration entre une Corée divisée et une Allemagne divisée. Elle mêlait des travaux d'art plastique (installations) à des pièces documentaires (vidéos et documents sur l'identité des personnes) en s'appuyant sur les recherches sur le postcolonialisme et les études sur le genre. C'était perceptible dans la narration de l'histoire des travailleurs coréens "invités" en Allemagne et dans la représentation picturale de la division du travail en fonction du genre.

Il s'agissait d'une exposition complexe et soigneusement élaborée, témoignant en outre d'une continuité avec des concepts et des travaux réalisés en Allemagne au 
cours de ces dernières années. Sun-Ju Choi, l'une de ses curatrices, a confirmé qu'avec un autre membre du comité de Korientation elle avait déjà participé à Projekt Migration (2005-2006). Cette exposition, la plus grande sur le thème de la migration en Allemagne, s'appuyait fortement sur l'art contemporain comme moyen d'expression. Son impact a été considérable parce qu'elle rassemblait des acteurs de disciplines très différentes. En témoigne son énorme catalogue où la sélection internationale d'auteurs représente les disciplines suivantes : sociologie, histoire, études post-coloniales, études de genre et beaux-arts. Il présente en outre des ceuvres d'art et des documents liés à la migration. Les textes sont publiés dans les langues originales, accompagnés de traductions. Le catalogue de Projekt Migration montre des similarités avec des revues d'avant-garde comme Documents, October ou Lettre internationale.

Néanmoins, Projekt Migration, qui s'est déroulé dans l'espace urbain ouvert de la ville de Cologne, a pâti du manque de participation d'“acteurs non allemands" au sein de la société allemande ou, pour reprendre le langage politique ordinaire, d'acteurs “issus de l'immigration”. C'est ce que déclara Sun-Ju Choi au cours de notre conversation du 23 novembre 2009. Il semble bon de s'attarder sur ce point parce que ce problème revient régulièrement lors d'entretiens avec des acteurs "non allemands", et qu'il ne fera que s'intensifier au cours des années à venir. Les membres de Korientation s'étaient sentis sous-représentés à l'époque de la conception de Projekt Migration, ce qui allait être l'un de leurs thèmes d'inspiration pour leur propre exposition. Selon Sun-Ju Choi, bien qu'ils aient joué le rôle de chercheurs scientifiques dans l'exposition, les véritables décisions - choix des matériaux historiques, "look" de l'exposition - étaient revenues aux "Mehrheitsgesellschaft", les membres de la majorité sociale allemande.

\section{De l'histoire au musée, la place renouvelée des migrants}

Ainsi, Partagés. Divisés. Unis peut être décrit comme un projet de "continuité dans la différence ${ }^{(12)}$ ", puisqu'il est issu du projet "allemand" Projekt Migration, dont il reprend les stratégies de représentation et les cadres conceptuels au profit de ses propres narrations, tout en développant ses différences et spécificités. Comme le soulignait Sun-Ju Choi, Partagés. Divisés. Unis a été conçu uniquement par des Germano-Coréens, tous issus du champ des études post-coloniales et de sa contrepartie naturelle, les études de genre. Cette exposition intégrait bien plus les conceptions post-coloniales, les perspectives de genre et les installations que Projekt Migration, et était en outre une dénonciation du manque de participation précédent et une affirmation de la différence. 
Pour finir, deux observations encore. Tout d'abord, Partagés. Divisés. Unis a été inauguré lors de la commémoration du vingtième anniversaire de la chute du Mur de Berlin, ce qui peut être interprété comme un signe de l'engagement des associations d'immigrés à représenter leur histoire et leurs souvenirs dans le cadre de commémorations nationales, par la participation à des commémorations de l'Histoire avec un grand H. La seconde observation est que les activistes politiques et les représentants des associations de migrants rencontrés ont non seulement des affinités avec les pensées post-coloniales et de genre, mais s'appuient explicitement sur des mouvements sociaux en provenance des États-Unis, comme le mouvement des droits civiques et l'élection d'Obama, perçus comme des actes cruciaux de la représentation politique. Leur travail se fonde donc résolument sur des liens transatlantiques et des réseaux transnationaux.

Dans le domaine de la représentation, ces liens s'expriment par le biais des arts plastiques et de la performance, pour construire des narrations "entre deux". C'est donc ainsi que les acteurs immigrés (non allemands) ont entrepris de représenter la migration, leur communauté et leurs liens transnationaux, et de gagner en puissance en devenant à leur tour des acteurs de la scène artistique. Ce dernier exemple révèle comment des groupes de migrants ayant un désir corporel de montrer leur histoire et leur biographie peuvent s'impliquer dans des institutions démocratiques, construire une "majorité" et ouvrir la démocratie en en modifiant les règles et en mettant en scène la migration hors de la pensée dominante.

Traduit de l'anglais par Françoise Bouillot

\section{Conférence}

- Gayatri Spivak, "Aesthetic Education in the Era of Globalisation", conférence donnée à la Freie Universität zu Berlin (Université libre de Berlin), Centre for Area Studies, History and Cultural Studies, 10 juin 2010, Berlin.

\section{Site Internet}

- Lien vers l'exposition Villa Global: http://www.villaglobal.de/

\section{Entretiens (par ordre chronologique)}

- John Durumba, directeur de l'association nigériane Ikuku-Berlin, Berlin, 9 novembre 2009.

- Sun-Ju Choi, membre fondateur de Korientation, Berlin, 23 novembre 2009.

- Martin Düspohl, directeur du Bezirksmuseum Friedrichshain-Kreuzberg, Berlin, 27 novembre 2009.

- Peter Junge, directeur du département africain de l'Ethnologisches Museum, Berlin, 22 avril 2010.

- Elena Brandalise, membre fondateur de Migrationsrat Berlin-Brandenburg e.V., Berlin, 28 avril 2010.

- Petra Zwacka, directeur du Jugendmuseum Schöneberg, Berlin, 11 août 2010.

- Curateurs et personnel du Jugendmuseum Schöneberg : M. Dubinina, M. Bahadoran, Layla, Yücel, Berlin, avril-août 2010. 


\section{Bibliographie}

- Jan Assmann, Das kulturelle Gedächtnis, Munchen, C.H. Beck, 2007.

- John Beverley, Subalternity and Representation. Arguments in Cultural Theory, Durham, Duke University Press, 2004.

- Beate Binder, Streitfall Stadtmitte. Der Berliner Schlossplatz, Köln, Böhlau Verlag, 2009.

- Pascal Blanchard, Gilles Boetsch, Éric Deroo, Sandrine Lemaire (dir.), Zoos humains. XIXe et XXe siècles, Paris, La Découverte, 2002.

- Dipesh Chakrabarty, "Museums in late democracies", in Humanities Research, vol. IX, n 1, 2002.

- Paul Connerton, How Societies Remember, Cambridge, Cambridge University Press, 1989.

- François Cusset, French Theory: Foucault, Derrida, Deleuze \& Cie et les mutations de la vie intellectuelle aux États-Unis, Paris, La Découverte, 2003.

- Éric Fassin, "La chaire et le canon. Les intellectuels, la politique et l'Université aux États-Unis", in Annales. Économies. Sociétés. Civilisations, n 2, mars-avril 1993.

- Ramón Grosfoguel, "Los dilemas de los estudios étnicos estadounidenses: multiculturalismo identitario, colonización disciplinaria y epistemologías decoloniales", in Universitas Humanística, n 63, 2007, pp. 35-47.

- Maurice Halbwachs, La Mémoire collective, Paris, Albin Michel, 1997 [1950].

- Wolfgang Kaschuba, "Deutsche Wir-Bilder nach 1945: Ethnischer Patriotismus als kollektives Gedächtnis?", in Jörg Baberowski, Hartmut Kaelble, Jürgen Schriewer (dir.), Selbstbilder und Fremdbilder. Repräsentation sozialer Ordnungen im Wandel, Frankfurt, Campus, pp. 295-329.

- Wolfgang Kaschuba, Einführung in die Europäische Ethnologie, München, Beck, 2006. Regina Römhild, "Alte Träume, neue Praktiken : Migration und Kosmopolitismus an den Grenzen Europas", in Transit Migration Forschungsgruppe (dir.), Turbulente Ränder. Neue Perspektiven auf Migration an den Grenzen Europas, Bielefeld, Transkript Verlag, 2007, pp. 211-222.

\section{Notes}

1. François Cusset, French Theory: Foucault, Derrida, Deleuze \& Cie et les mutations de la vie intellectuelle aux États-Unis, Paris, La Découverte, 2003.

2. Éric Fassin, "La chaire et le canon. Les intellectuels, la politique et l'Université aux États-Unis", in Annales. Économies. Societés. Civilisations, n 2, mars-avril 1993 ; John Beverley, Subalternity and Representation. Arguments in Cultural Theory, Durham, Duke University Press, 2004.

3. Wolfgang Kaschuba, Einführung in die Europäische Ethnologie, München, Beck, 2006.

4. Ramón Grosfoguel, "Los dilemas de los estudios étnicos estadounidenses: multiculturalismo identitario, colonización disciplinaria y epistemologías decoloniales", in Universitas Humanística, n 63, 2007, pp. 35-47.

5. Dipesh Chakrabarty, "Museums in Late Democracies", in Humanities Research, vol. IX, nº 1, 2002.

6. Le directeur du musée du quartier de Friedrichshain-Kreuzberg, Martin Düspohl, m'avait également dit en novembre 2009 que l'exposition Ein jeder nach seiner Façon? 300 Jahre Zuwanderung nach Friedrichshain-Kreuzberg

("Chacun/chacune à sa façon ? 300 ans de migration à Friedrichshain-Kreuzberg") allait fermer malgré son succès.

7. Proche de l'île aux musées qui regroupe des collections européennes provenant des plus anciennes civilisations d'Asie Mineure et d'Égypte jusqu'aux ceuvres du XIX siècle, le château accueillera les collections des arts et cultures extra-européennes. L'ensemble formera un "Humboldt-Forum" réactualisant la vieille idée des Lumières de faire du centre de Berlin une "terre libre des Arts, des Sciences et de la Communication"(N. d. T.).

8. Ikuku-Berlin a pour objectif de promouvoir la culture nigériane à Berlin. Elle est apparue en 2006 comme une initiative germano-nigériane.

9. Cette façon de réduire la "migration" au silence est un élément constitutif du processus de construction de la nation allemande. Kaschuba décrit comment la dramatique expérience de la migration a été assimilée et conçue comme une "altérité". Les problèmes psychologiques et sociaux qui lui sont associés sont eux aussi passés sous silence, de sorte que se souvenir devient souvent tabou, et la mémoire, un traumatisme. Voir Wolfgang Kaschuba, Einführung in die Europäische Ethnologie, München, Beck, 2006, p. 310. Ce processus de silence - où la migration et la transformation sont jugées honteuses - se perçoit dans les "diasporas allemandes", les Gastarbeiter (travailleurs "invités"), et "l'intégration" de l'ancienne RDA après la "réunification" (ibid., pp. 295 - 329).

10. Beate Binder, Streitfall Stadtmitte. Der Berliner Schlossplatz, Köln, Böhlau Verlag, 2009, p. 292.

11. La $N G B K$ est financée par la Stiftung Deutsche Klassenlotterie depuis 1969.

12. Cette expression est reprise de Gayatri Spivak (conférence donnée au FU Berlin, juin 2010). 